\title{
Uma discussão sobre as diferentes classificações da estratégia de postponement
}

\section{A discussion on the different classifications of the postponement strategy}

\author{
Karine Araujo Ferreira \\ Universidade Federal de Ouro Preto \\ Departamento de Engenharia de Produção- DEENP/ICEA \\ karine@deenp.ufop.br \\ Rosane Lúcia Chicarelli Alcântara \\ Universidade Federal de São Carlos \\ Departamento de Engenharia de Produção- DEP/UFSCar \\ rosane@dep.ufscar.br
}

Resumo A estratégia de postponement é cada vez mais utilizada por empresas preocupadas em gerenciar a complexidade e a variedade crescente de produtos. Em sua essência, o postponement consiste em adiar o máximo possível qualquer deslocamento e/ou configuração final de produtos até que a demanda seja conhecida. Nos últimos anos, este tema tem despertado o interesse de acadêmicos e executivos em diversos países. Apesar da atenção crescente ao tema, poucos trabalhos abordam as diferenças conceituais entre as diversas classificações de postponement apresentadas em mais de 60 anos de literatura sobre o tema e em diferentes áreas de pesquisa. Assim, neste artigo procurar-se-á investigar e apresentar essas classificações, identificando suas semelhanças e diferenças. A abordagem de pesquisa realizada é a qualitativa, sendo o método caracterizado como teórico-conceitual, voltado à busca e à revisão da literatura sobre a estratégia de postponement.

Palavras-chave: POSTERGAÇÃo, CUSTOMIZAÇÃo EM MASSA, GESTÃO DA CADEIA DE SUPRIMENTOS.

ABSTRACT The postponement strategy has been increasingly used by companies dealing with the increasing complexity and variety of products. In essence, postponement is used to delay as much as possible the final configuration and/or movement of products, until the demand is known. In recent years, this subject has drawn the attention of scholars and executives in several countries. Despite the increasing attention to the subject, few studies discuss the conceptual differences between the various classifications of postponement presented in more than 60 years of literature on this topic in different research areas. Thus, this article will investigate these classifications, presenting their similarities and differences. This study used a qualitative 
approach and the theoretical-conceptual method focused on the search and review of literature on postponement strategy.

Keywords: POSTPONEMENT, MASS CUSTOMIZATION, SUPPLY CHAIN MANAGEMENT.

\section{INTRODUÇÃO}

Um dos principais desafios das empresas modernas consiste em produzir exatamente o que os consumidores desejam, no local apropriado, com custo e tempo almejados (GRAMAN; MAGAZINE, 2006; WIKNER et al., 2007). Para Van Hoek et al. (1999), uma maneira de enfrentar este desafio consiste em obter benefícios pela economia de escala para determinados estágios da produção de produtos, deixando a etapa de diferenciação para o ponto em que a demanda é conhecida. Envolve também a implementação de estratégias de estoque específicas, em que produtos sejam estocados em armazéns centralizados até o momento em que o pedido é realizado. Esta estratégia é conhecida como postponement e consiste em adiar o máximo possível qualquer movimentação e/ou configuração final de produtos (VAN HOEK, 2001). Um exemplo clássico da aplicação dessa estratégia pode ser verificado na fabricação de tintas. Atualmente, por meio de parceria estabelecida entre produtores e lojas de tintas, o ponto de diferenciação do produto transfere-se para dentro da loja e para o ponto de venda (mais próximo do consumidor), ou seja, os produtores vendem às lojas as bases de tinta, geralmente na cor branca, e os pigmentos de outras cores. Assim, quando o cliente faz o pedido de determinada cor, a loja de tinta faz sua configuração final, misturando a base e o pigmento para fabricar a cor desejada pelo cliente, eliminando, assim, a necessidade de estoque de cada cor de tinta e a obsolescência do produto (PIRES, 2004).

A Dell é outro exemplo da aplicação dessa estratégia, pois é uma empresa que fornece produtos personalizados em poucos dias, a preços compatíveis com um sistema de produção em massa. Para isto, compra módulos e somente monta os produtos finais quando os pedidos dos clientes são realizados (SAMPAIO, 2003; PIRES, 2004). A montadora General Motors Brasil segue o mesmo caminho, atende a $70 \%$ da demanda dos veículos da marca Celta pela internet e os entrega entre 4 e 10 dias aos consumidores, a partir de quatro centros de distribuição (SAMPAIO, 2003) . No setor alimentício, esta estratégia é também observada por Ferreira e Alcântara (2011), que investigaram a aplicação do postponement em três das principais processadoras de suco de laranja concentrado e congelado no Brasil. Estas empresas têm sua produção majoritariamente voltada para a exportação. Nela, toda a etapa de abastecimento e produção do suco concentrado e congelado é feita sob previsão (para estoque e em formulação praticamente única). Esse suco é transportado para os países de destino, onde o suco pode, então, ser customizado de acordo com a embalagem, volume, diluição, adição de açúcar, aroma etc., conforme exigências do cliente local. Esta customização geralmente é realizada por uma empresa independente (situada no exterior), parceira no processo de distribuição e cliente das empresas de suco brasileiras.

Estes exemplos mostram algumas das possíveis aplicações do postponement, que não é um conceito novo ou apenas um conceito teórico. O assunto vem sendo estudado de forma descontinuada há décadas. O termo foi introduzido pela primeira vez na literatura em 1950 
(ALDERSON, 1950) e as primeiras experiências utilizando o postponement remontam à década de 1920 (CLM, 1995). Porém, somente com o desenvolvimento recente da tecnologia, especialmente as tecnologias de informação e comunicação, de transporte e de produção, que o conceito tem se tornado mais atraente e sua aplicação, viável.

Nos últimos anos, este tema tem despertado o interesse de acadêmicos e executivos em diversos países. Trabalhos abordando o conceito podem ser encontrados em diferentes áreas, como logística, produção, marketing, gestão de operações e gestão da cadeia de suprimentos, conforme destacado por Van Hoek (2001) e Yang et al. (2004a). Em determinados estudos, observa-se que o postponement apresenta-se como uma das iniciativas e práticas na gestão da cadeia de suprimentos (PIRES, 2004).

Apesar da atenção crescente ao tema, poucos trabalhos abordam as diferenças conceituais e as semelhanças entre as diferentes classificações da estratégia de postponement surgidas ao longo de mais de 60 anos de pesquisa sobre o tema. Adicionalmente, poucas pesquisas buscam analisá-las e discuti-las de maneira abrangente. Assim, neste artigo procurar-se-á investigar e definir as diferentes classificações de postponement presentes em artigos de áreas de pesquisa distintas, apresentando suas semelhanças e diferenças.

Neste sentido, este trabalho tem o propósito de corroborar para uma melhor compreensão da estratégia de postponement, podendo ser uma importante ferramenta para que profissionais de empresas e pesquisadores possam aplicar a estratégia mais adequadamente.

A pesquisa realizada neste trabalho é de natureza qualitativa, com base em uma revisão bibliográfica aprofundada sobre o tema. Essa revisão permitiu analisar as diferentes classificações de postponement existentes na literatura.

A seguir são apresentados os principais conceitos e classificações de postponement identificados na revisão de literatura, discutindo-se essas classificações, suas semelhanças e diferenças.

\section{PostPoneMENT: DEFINIÇÕES E CLASSIFICAÇÕES}

O conceito de postponement foi introduzido pela primeira vez na literatura de marketing por Alderson (1950) como uma maneira de mudar a forma, identidade ou lugar de produtos em um momento tão tardio quanto possível dentro dos processos de manufatura e distribuição física. Operacionalmente, isto envolve projetar e desenvolver produtos padrão ou genéricos que possam ser customizados rapidamente e a baixo custo, uma vez que a demanda do consumidor seja conhecida. Envolve também a implementação de estratégias de estoque específicas, permitindo produtos em um único local no canal de distribuição até o momento em que o pedido é realizado. Postergar a movimentação do produto foi denominado "postponement de tempo" (time postponement), enquanto a postergação na diferenciação do produto foi denominada "postponement de forma" (form postponement).

Zinn e Bowersox (1988) definem o postponement como uma maneira de mudar para um sistema orientado à demanda, uma vez que esta estratégia permite atrasar a movimentação ou formulação final de produtos tipicamente produzidos em massa até que os pedidos dos consumidores sejam recebidos. Eles ampliaram as alternativas do postponement e propuseram que o conceito poderia ser separado em cinco diferentes tipos, quatro relacionados com alterações 
de forma do produto (etiquetagem, embalagem, montagem e manufatura) e o quinto relacionado ao tempo (centralização dos estoques).

Para Cooper (1993), o conceito pode ser visto como um determinante da estratégia de distribuição global de uma empresa. Quanto à classificação, o autor especifica quatro possíveis estruturas para as operações finais de manufatura, as quais dependem do tipo de operações finais realizadas, do tipo de produto e do mercado. Três características operacionais básicas foram utilizadas para promover a classificação: marca, formulação e periféricos dos produtos. As quatro estruturas são:

- manufatura centralizada (unicentric postponement) - sistema de manufatura centralizada, em que a fabricação é integrada a uma planta global e a distribuição é feita por encomenda. Ajusta-se a casos em que as três características do produto são as mesmas em todos os mercados onde o produto é vendido, ou seja, são padronizadas. Assemelha-se ao postponement de tempo;

- manufatura agrupada (bundled manufacturing) - ideal para produtos com marca global e formulações diferentes em cada mercado, como os aparelhos de TV. Nessa alternativa, a diferenciação do produto é retardada no processo produtivo (na fábrica) e o produto semi-elaborado é despachado e mantido em localização centralizada (armazém ou centro de distribuição). Essa estratégia é a combinação do postponement de forma com o de tempo;

- montagem postergada (deferred assembly) - sistema de montagem adiada, em que a produção é puxada e a manufatura final dos produtos acontece nos centros de distribuição locais. Adequado para produtos com uma marca global, com periféricos diferenciados e formulações diferenciadas. Assemelha-se aos postponements de montagem e manufatura descritos anteriormente;

- embalagem postergada (deferred packaging) - ideal para quando o rótulo e/ou a embalagem são diferenciados conforme o mercado. Relaciona-se aos postponements de etiquetagem e embalagem de Zinn e Bowersox (1988).

Bowersox e Closs (1996) destacam dois tipos de postponement. o postponement de manufatura (ou forma) e o postponement logístico (de tempo). O postponement de manufatura consiste em fabricar um produto base ou padrão em quantidades suficientes para realizar economia de escala, enquanto as características de finalização são adiadas até que os pedidos dos consumidores sejam recebidos. Já o postponement logístico consiste em manter toda linha de produtos já acabados em estoque centralizado. O deslocamento dos estoques é adiado até o recebimento do pedido dos clientes. Quando a demanda ocorre, os pedidos são transportados diretamente ao varejo ou ao consumidor.

Van Hoek (1999) conceitua postponement como uma maneira de atrasar ou postergar o ponto de diferenciação do produto, isto é, atrasar os processos em que os produtos são transformados em especificações únicas para o consumidor. Este mesmo autor define também, além do postponement de forma e tempo, o postponement de lugar. Para ele, o postponement de lugar 
implica estocar os produtos acabados em localizações centrais, antes de a demanda ocorrer (atrasando a determinação do local), enquanto o postponement de tempo consiste em atrasar a movimentação de produtos finais efetuando-a apenas sob pedido (atrasando a determinação de tempo). Percebe-se que para Van Hoek (1999) o postponement logístico engloba o postponement de tempo e o de lugar, ou seja, a combinação de postponement de tempo e lugar é entendida como postponement logístico.

Autores como Feitzinger e Lee (1997) definem o postponement como um método necessário para uma empresa realizar a estratégia de customização em massa e propõem a divisão do postponement de forma em duas diferentes esferas: postponement do produto e postponement do processo. No postponement de produto, a empresa projeta o produto usando estrutura modular ou componentes padronizados para adiar a diferenciação dos produtos. No postponement dos processos, os processos de produção e distribuição são projetados para permitir a diferenciação do produto à jusante na cadeia de suprimentos.

Uma importante contribuição é feita por Pagh e Cooper (1998), que combinaram quatro estratégias de postponement para a cadeia de suprimentos em uma matriz 2x2 (Figura 1). Na matriz, o postponement de forma é chamado de postponement de manufatura (manufacturing postponement) e o postponement de tempo é chamado de postponement logístico (logistics postponement). As quatro estratégias são então desenvolvidas pela combinação do postponement de manufatura e logística. As duas primeiras estratégias são representadas tanto pelo postponement de manufatura como pelo postponement logístico. As outras duas são formadas pela combinação de ambas, denominada estratégia de postponement total ou pleno, e pela ausência de ambas, denominada estratégia de especulação total.

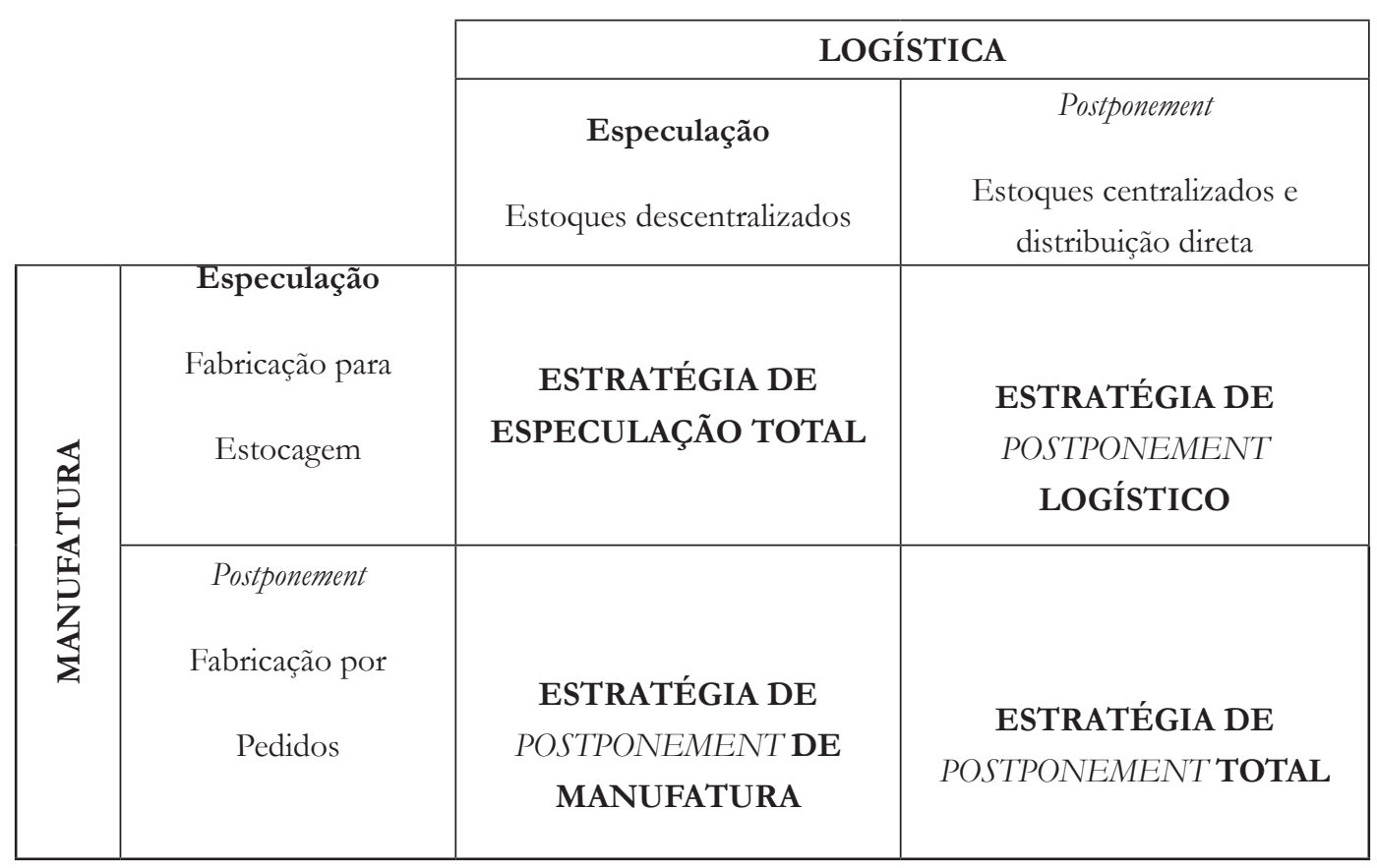

Figura 1. MATRIZ POSTPONEMENT/ESPECULAÇÃO E ESTRATÉGIAS DA CADEIA DE SUPRIMENTOS

Fonte: Adaptado de Pagh e Cooper (1998) 
Para incluir a aplicação do postponement em toda a extensão da cadeia de suprimentos, Waller et al. (2000) consideram mais apropriada a nomenclatura dada a partir dos estágios em que ele pode ocorrer, bem como seu envolvimento com os diferentes membros da cadeia de suprimentos. Assim, tais estratégias de postponement propostas pelos autores incluem o postponement upstream, postponement downstream e postponement de distribuição. O postponement upstream (montante) ocorre com o retardo de ordens de materiais dos fornecedores até que se tenha a informação de pedidos. É apropriado para empresas que empregam matérias-primas caras e para as que produzem a partir de projetos, como os construtores de reatores nucleares. Já o postponement downstream (jusante) é a postergação que agrega valor próximo da demanda e é executada pela empresa mais próxima do consumidor final (não necessariamente por quem produziu a primeira etapa). Este tipo de postponement pode ser definido como o atraso de algum tipo de mudança física no produto, após o estágio primário de manufatura, tais como manufatura adicional, adição de características, ou adição de alguma função que adicione valor para uma cadeia de suprimentos específica. Os autores mencionam também o postponement de distribuição (similar ao de lugar), que deve ocorrer depois que o produto já estiver com as configurações finais para ser consumido.

Apesar de Waller et al. (2000) já mencionarem a aplicação do postponement na cadeia de suprimentos no ano de 2000, apenas recentemente o conceito tem sido aplicado mais extensivamente à gestão da cadeia de suprimentos, promovendo uma nova maneira de pensamento sobre o projeto do produto, projeto do processo e gestão da cadeia de suprimentos, sendo seu uso determinado por sua estrutura e suas características. A aplicação do postponement afeta a estruturação da cadeia de suprimentos e frequentemente guia sua reconfiguração (YANG et al., 2004b). Ampliando a extensão de onde e quando é aplicado, várias estratégias de postponement têm sido propostas, como as apresentadas pelos autores, que incluem o postponement de desenvolvimento do produto e o postponement de compras aos já mencionados postponement de produção e logístico. Segundo Yang et al. (2004b), o escopo das duas últimas estratégias é restrito por assumir que: 1) o postponement de produção difere do de compras, no qual é necessário que matérias-primas e componentes tenham sido comprados para iniciar a produção antecipada de pedidos; (2) o postponement logístico é aplicado somente quando há produtos acabados. Os mesmos autores definem o postponement de desenvolvimento do produto como uma oportunidade para reduzir lead times de projeto e reprojetos custosos. Por focar inicialmente em decisões de especificações do produto, que provavelmente são padronizadas e possuem poucas modificações, decisões de projetos sobre partes menos conhecidas e previsíveis do produto podem ser postergadas até que a melhor informação esteja disponível. Já o postponement de compras permite postergar a compra de componentes ou matérias-primas até que a demanda seja conhecida, eliminando o risco de produto obsoleto em estoque. Esta estratégia, em conjunto com o postponement de produção, permite à empresa evitar estoques de produtos acabados em antecipação a pedidos futuros. O postponement de compras é mais adequado para componentes e matérias-primas caros e frágeis e que vêm em diferentes tamanhos e divisões. Exemplo: reatores nucleares.

Por fim, Garcia-Dastugue e Lambert (2007) destacam que o postponement não deve ser implementado sem considerar o impacto para outros membros da cadeia de suprimento e 
introduzem o conceito de postponement interorganizacional, ou seja, consideram a aplicação do postponement em toda a extensão da cadeia de suprimentos. Para eles, há situações nas quais múltiplos membros da cadeia de suprimento são capazes de postergar as atividades até que o consumidor final realize o pedido. Adicionalmente, eles diferenciaram os tipos de postponement pela mudança ou não na sequência de atividades. O postponement pela mudança na sequência de atividades corresponderia ao postponement de forma e é implementado por mudar o projeto de produto, os processos de manufatura e a estrutura da cadeia de suprimentos, ou seja, os produtos e os processos são redesenhados usando a padronização, projeto modular ou reestruturação de processos. Por outro lado, o postponement pode ser implementado sem mudar a sequência de atividades, ou seja, sem mudar o projeto do produto, o processo de manufatura ou a estrutura da cadeia de suprimentos e é denominado pelos mesmos autores como postponement baseado no tempo. O postponement baseado no tempo refere-se ao atraso intencional de atividades ao tempo mais tarde e não requer mudança no local onde o trabalho é feito, isto é, o ponto de diferenciação do produto não é mudado.

\section{DISCUSSÃO SOBRE OS CONCEITOS E CLASSIFICAÇÕES DE POSTPONEMENT}

Analisando as definições descritas, verifica-se que apesar da vasta literatura sobre o postponement produzida em mais de 60 anos de estudo sobre o tema, a definição original ainda permanece atual. Ou seja, apesar das diferentes conceituações e áreas de aplicação do conceito surgidas no decorrer dos anos, a ideia inicial proposta por Alderson em 1950 não sofreu grandes modificações. As definições mais recentes adicionam a esta definição inicial as possíveis aplicações do postponement, por exemplo, sua adoção para possibilitar a customização em massa. Além disso, estudos de tipologias da estratégia de retardo advindos de diferentes correntes de pesquisa foram desenvolvidos, bem como análises de custos, resultados e sua adequabilidade à cadeia de suprimentos. Com a introdução do conceito de gestão cadeia de suprimentos, o postponement, que era abordado principalmente entre duas empresas ou em parte da cadeia, passa também a ser discutido por vários autores em um sentido mais amplo, abrangendo toda a extensão da cadeia de suprimentos.

Apesar do conceito de postponement não ter sofrido grandes alterações ao longo dos anos, sua aplicação tornou-se mais viável e crescente em diferentes setores, em virtude do desenvolvimento da tecnologia, especialmente as tecnologias de informação e comunicação, de transporte e de produção.

Já em relação às classificações de postponement, o Quadro 1 sintetiza os tipos apresentados por cada autor. É possível visualizar neste quadro que, embora diferentes classificações e denominações de postponement tenham sido elaboradas pelos autores ao longo dos anos, grande parte delas tem o mesmo significado, sendo as classificações de postponement de tempo e de forma propostas originalmente por Alderson (1950) os principais tipos estudados. Muitas das classificações citadas pelos diversos autores podem ser consideradas subcategorias, ou novas denominações para os postponements de forma e tempo. Os postponements de etiquetagem, embalagem, manufatura e rótulo, por exemplo, são definidos por Bowersox e Closs (1996) como subcategorias ou atividades nas quais o postponement de forma pode acontecer. Assim, além do 
tipo de postponement (forma ou tempo, por exemplo), é preciso definir quais atividades serão postergadas, ou seja, fabricação, embalagem, montagem, etiquetagem, rótulo, distribuição etc.

Quadro 1. Síntese das classificações de postponement

\begin{tabular}{|c|c|}
\hline Autor & Classificações de Postponement \\
\hline Alderson (1950) & $\begin{array}{l}\text { Postponement de forma } \\
\text { Postponement de tempo }\end{array}$ \\
\hline $\begin{array}{l}\text { Zinn e Bowersox } \\
(1988)\end{array}$ & $\begin{array}{l}\text { Postponement de forma é subdividido em } 4 \text { tipos: } \\
\text { de etiquetagem } \\
\text { de embalagem } \\
\text { de montagem } \\
\text { de fabricação } \\
\text { Postponement de tempo }\end{array}$ \\
\hline Cooper (1993) & $\begin{array}{l}\text { Manufatura centralizada }(=\text { de tempo }) \\
\text { Manufatura agrupada }(=\text { de tempo }+ \text { de forma }) \\
\text { Montagem postergada (= de montagem }+ \text { de manufatura }) \\
\text { Embalagem postergada (= de etiquetagem }+ \text { de embalagem })\end{array}$ \\
\hline $\begin{array}{l}\text { Bowersox e Closs } \\
(1996)\end{array}$ & $\begin{array}{l}\text { Postponement de manufatura (= de forma) } \\
\text { Postponement logístico (= de tempo }+ \text { de lugar })\end{array}$ \\
\hline Van Hoek (1999) & $\begin{array}{l}\text { Postponement de forma } \\
\text { Postponement de tempo } \\
\text { Postponement de lugar }\end{array}$ \\
\hline $\begin{array}{l}\text { Feitzinger e Lee } \\
(1997)\end{array}$ & $\begin{array}{l}\text { Postponement de forma é subdividido em: } \\
\text { Postponement de produto } \\
\text { Postponement de processo }\end{array}$ \\
\hline Pagh e Cooper (1998) & $\begin{array}{l}\text { Especulação total } \\
\text { Postponement de manufatura (= de forma) } \\
\text { Postponement de logística (= de tempo + de lugar) } \\
\text { Postponement total }\end{array}$ \\
\hline Waller et al. (2000) & $\begin{array}{l}\text { Postponement upstream } \\
\text { Postponement downstream (= postponement de forma) } \\
\text { Postponement de distribuição (= postponement de lugar) }\end{array}$ \\
\hline Yang et al. (2004b) & $\begin{array}{l}\text { Postponement de projeto do produto } \\
\text { Postponement de compras (= postponement upstream) } \\
\text { Postponement de produção (= de forma) } \\
\text { Postponement logístico (= de tempo }+ \text { de lugar) }\end{array}$ \\
\hline $\begin{array}{l}\text { Garcia-Dastugue e } \\
\text { Lambert (2007) }\end{array}$ & $\begin{array}{l}\text { Postponement pela mudança na sequência das atividades }(=\text { de forma }) \\
\text { Postponement baseado no tempo (= de tempo) }\end{array}$ \\
\hline
\end{tabular}

Fonte: Elaborada pela autora. 
Adicionalmente, pode-se destacar que além das atividades que podem ser postergadas deve-se destacar que o postponement pode ocorrer em diferentes estágios da cadeia de suprimentos. De acordo com Cardoso (2002), estágio é o local da cadeia de suprimentos no qual a diferenciação do produto pode acontecer. A mesma autora destaca os seguintes estágios da cadeia de suprimentos em que a postergação pode ser realizada: da planta fabril para um fornecedor upstream, entre plantas, de um depósito para uma planta fabril, de um cliente downstream para uma planta fabril ou para um depósito; e de depósito para depósito. Corroborando com esta discussão, cabe destacar os autores Lee e Billington (1994), que sugeriram que o postponement pode ocorrer cedo ou tarde na cadeia de suprimentos. O postponement de tempo é antecipado quando o estoque está centralizado na planta fabril e tardio quando sua localização encontra-se no armazém, centro de distribuição ou em outro intermediário do canal. Já a postergação de forma é antecipada quando a diferenciação do produto ocorre na planta fabril. Nesse caso, o processo de produção ou o próprio produto é reprojetado para adiar a diferenciação num ponto avançado no processo de produção, dentro da planta fabril. Finalmente, a postergação de forma é tardia quando o ponto de diferenciação de produto é transferido para uma localização próxima ao consumidor.

Em síntese, é possível verificar que o postponement pode, então, ocorrer internamente em uma mesma planta fabril ou ao longo de uma cadeia de suprimentos. No primeiro caso, adia-se somente o momento em que certas etapas da produção são realizadas, mantendo-se as operações produtivas no mesmo local, enquanto, no segundo caso, as atividades de produção são postergadas para outros membros da cadeia de suprimentos, ou seja, a postergação pode ocorrer em locais diferentes daquele onde foram executadas as atividades primárias de manufatura, como ocorre no exemplo da produção de tintas, já mencionado.

\section{CONSIDERAÇÕES FINAIS}

Este estudo buscou aprofundar a pesquisa sobre as diferentes classificações de postponement, destacando as diferenças e pontos comuns entre elas. Para cumprir este objetivo, foi realizada uma revisão da literatura existente sobre o tema.

Essa revisão teórica possibilitou verificar que várias classificações ou tipos de postponement foram criados ao longo dos anos. Apesar disso, verificou-se que muitas dessas classificações têm o mesmo significado, sendo muitas delas apenas um novo rótulo para as classificações de postponement já existentes, como os postponements de forma e tempo, já definidos por Alderson em 1950. Acredita-se que a existência desses diferentes rótulos ocorre porque ao longo de mais de 60 anos de pesquisa sobre o tema, artigos sobre postponement despontaram não somente na literatura de logística e marketing, em que o tema tradicionalmente aparecia, mas também nas áreas correlatas de engenharia, administração de empresas, gestão de operações, projeto de produto e gestão da cadeia de suprimentos.

Embora a definição do tema não tenha sofrido grandes alterações ao longo dos anos, sua aplicação prática tem se tornado cada vez mais atraente e adotada por empresas de diferentes setores, tais como automotivo, têxtil, tintas, eletrônicos, alimentício e outros.

É importante também ressaltar que a estratégia de postponement pode ser aplicada em diferentes processos ou partir de diferentes atividades de desenvolvimento de um produto (pro- 
jeto, compras, operações e fabricação, manufatura final, montagem, embalagem, etiquetagem/ rótulo e distribuição). Adicionalmente, o postponement pode ocorrer em diferentes posições na cadeia de suprimentos, ou seja, à montante ou à jusante a uma empresa ou, ainda, internamente, na própria planta fabril. Assim, estes três fatores (tipo de postponement, atividade a ser postergada e posição na cadeia de suprimentos onde acontecerá a postergação) devem ser cuidadosamente analisados para escolha do tipo de postponement mais adequado às características da empresa e do produto que está sendo fabricado.

Por fim, cabe ressaltar a importância do postponement como estratégia para que as empresas possam simultaneamente reduzir custos e atender às especificações individuais dos clientes. Assim, pesquisas adicionais sobre a aplicação dos diferentes tipos de postponement em diferentes setores industriais devem ser realizadas.

\section{ReferÊNCIAS}

ALDERSON, W. Marketing efficiency and the principle of postponement. Cost and Profit Outlook, n. 3, p. 15-18, 1950.

BOWERSOX, D. J.; CLOSS, D J. Logistical management. the integrated supply chain process. New York, NY: McGraw-Hill, 1996.

CARDOSO, P. A. O princípio da postergação: um estudo na cadeia de suprimentos das tintas para impressão. 2002. 158p. Tese (Doutorado em Engenharia de Produção) - Departamento de Engenharia Industrial da Pontíficia Universidade Católica do Rio de Janeiro, Rio de Janeiro.

COOPER, J. Logistics strategies for global business. International Journal of Physical Distribution and Logistics Management, v. 23, n. 4, p. 12-23, 1993.

COUNCIL OF LOGISTICS MANAGEMENT (CLM). World class logistics: the challenge of managing continuous change. Oak Brook: CLM, 1995.

FEITIZINGER, E.; LEE, H. L. Mass customization at Hewlett-Packard: the power of postponement. Harvard Business Review, v. 75, n. 1, p.116-121, 1997.

FERREIRA, K.A; ALCÂNTARA, R.L.C. Aplicação da estratégia de postponement em empresas processadoras de suco de laranja. In: SIMPÓSIO DE ENGENHARIA DE PRODUÇÃO, 18, 2011, Bauru. Anais eletrônicos... Disponível em: < http://www.simpep.feb.unesp.br/ anais_simpep.php>. Acesso em: jan.2012.

GARCIA-DASTUGUE, S.; LAMBERT, D. Interorganizational time-based postponement in the supply chain. Journal of Business Logistics, v.28, n.1, p.57-76, 2007.

GRAMAN, G. A.; MAGAZINE, M. J. Implementation issues influencing the decision to adopt postponement. International Journal of Operations \& Production Management, v. 26, n. 10, p. 1068-1083, 2006.

LEE, H. L.; BILLINGTON, C. Designing products and process for postponement. In: DASU, E.; EASTMAN, C. (Eds.). Management of design: engineering and management perspectives. Boston: Kluwer Academic Publishers, 1994. p. 105-122. 
PAGH, J. D.; COOPER, M. C. Postponement and speculation strategies: how to choose the right strategy. Journal of Business Logistics, v. 19, n. 2, p. 13-32, 1998.

PIRES, S. R. I. Gestão da cadeia de suprimentos: conceitos, estratégias, práticas e casos. São Paulo: Atlas, 2004.

SAMPAIO, M. O poder estratégico do postponement. 2003.198p. Tese (Doutorado em Administração) - Fundação Getúlio Vargas, São Paulo, 2003.

VAN HOEK, R. I.; PEELEN, E.; COMANDEUR, H. R. Achieving mass customization through postponement: a study of international changes. Journal of Market Focused Management, v. 3, p. 353-368, 1999.

VAN HOEK, R. I. Postponement and the reconfiguration challenge for food supply chains. Supply Chain Management, v. 4, n. 1, p. 18-34, 1999.

. The rediscovery of postponement: a literature review and directions for research. Journal of Operations Management, v. 19, n. 2, p.161-184, 2001.

WALLER, M. A.; DABHOLKAR, P. A.; GENTRY, J. J. Postponement, product, customization, and market-oriented supply chain management. Journal of Business Logistics, v. 21, n. 2, p. 133-156, 2000.

WIKNER, J.; NAIM, M. M.; RUDBERG, M. Exploiting the order book for mass customized manufacturing control systems with capacity limitations. IEEE Transactions on Engineering Management, v. 54, n. 1, p. 145-155, 2007.

YANG, B.; BURNS, N. D.; BACKHOUSE, C. J. Postponement: review and an integrated framework. International Journal of Operations \& Production Management, v. 24, n. 5, p. 268-487, 2004a.

. Management of uncertainty through postponement. International Journal of Production Research, v. 42, n. 6, p. 1049-1064, 2004b.

ZINN, W.; BOWERSOX, D. J. Planning physical distribution with the principle of postponement. Journal of Business Logistics, v. 9, n. 2, p. 117-136, 1988. 